\title{
A Comparison of Methods for Link Sign Prediction with Signed Network Embeddings
}

\author{
Sandra Mitrović \\ LIRIS \\ KU Leuven \\ Leuven, Belgium \\ sandra.mitrovic@kuleuven.be
}

\author{
Laurent Lecoutere \\ LIRIS \\ KU Leuven \\ Leuven, Belgium \\ laurent.lecoutere@student.kuleuven.be
}

\author{
Jochen De Weerdt \\ LIRIS \\ KU Leuven \\ Leuven, Belgium \\ jochen.deweerdt@kuleuven.be
}

\begin{abstract}
In many real-world networks, it is important to explicitly differentiate between positive and negative links, thus considering the observed networks as signed. To derive useful features, just as in the case of unsigned networks, representation learning can be used to learn meaningful representations of a network that characterize its underlying topology. Several methods for learning representations on signed networks have already been proposed but have not been systematically benchmarked together before. Hence, in this paper, we bridge this literature gap providing a quantitative and qualitative benchmark of the four most prominent representation learning methods for signed networks. Results on three different datasets for link sign prediction showcase the superiority of the StEM method over its competitors both from a predictive performance and runtime perspective.
\end{abstract}

Index Terms-Link Sign Prediction, Signed Network Embeddings, Signed Social Networks

\section{INTRODUCTION}

Networks are ubiquitous in real-life situations as they are a straightforward and transparent way to represent different kind of relations: whether it is to capture the interactions between proteins, relations between users on social media or collaborations between researchers, all this is possible with a network structure. Furthermore, depending on the particular scenario, the underlying formalism of a network can be adjusted as necessary. As such, if the direction of a relationship is relevant for the problem at hand, we can consider directed networks, if different types of entities (e.g. clients and merchants) are of the most importance, then bipartite networks can be applied, if tracking the relationships throughout the time is crucial, dynamic networks should be considered etc. In certain cases, attention is given to the entities (nodes), for the others, rather the relations/links (edges) between these entities are of interest. While edges can be characterized using different weights to denote some kind of intensity of the relationship (which leads to weighted networks), there might be scenarios where only the type of the edge plays a crucial role. For example, in a financial network it is important to distinguish regular from fraudulent transactions, in customer-product networks it is crucial to know if a customer has liked the product (by e.g. giving a positive review about it) while in a social network one might be interested to differentiate between "friends" and "foes". In these examples, relationships between entities are extremely polarized, so each relationship could be basically denoted by either a positive or a negative sign (that is, considered as either positive or negative links). Networks containing this kind of edges are known in the literature as signed networks.

This paper focuses on link sign prediction in the static signed networks, i.e., the problem of predicting the sign of a link in a signed network which is regarded as static. Despite a variety of methods available in the literature [10], [18], [21]-[24], our emphasis is on those methods that address this problem by means of representation learning on networks/graphs. Representation learning is a particular field in machine learning, that aims to find a representation (also known as an embedding) of the entity in a lower dimensional space while at the same time maximally preserving informative characteristics and existing variance in the data, in order to obtain useful representations which could, thereafter, be exploited as the features for different machine learning tasks [17]. More particularly, when applied on network data, representation learning provides a low-dimensional vector of different network elements (nodes/edges/subgraphs or even the entire graph) which characterize the underlying network topology and link structure as well as possible.

Applying existing representation learning methods developed for unsigned networks for the purpose of link sign prediction is not feasible since the former are typically based on a homophily hypothesis which claims that similar nodes are more likely to be connected. This is intrinsically not valid as in signed network connections might rather reveal dissimilarities ("foes" instead of "friends" or a dislike instead of a like). Therefore, it is necessary to develop representation learning methods on signed networks. In fact, several representation learning-based approaches have recently been designed to address the link sign prediction problem in static signed networks: SNE [1], SiNE [2], SIDE [3] and StEM [4]. Despite the relatively low number of techniques presented, surprisingly, none of the existing studies compares performances of all four of these methods. Furthermore, from the existing partial comparisons, it is not clear which method performs the best. For example, results from [4] show that StEM is outperforming SiNE and results from [3] showcase that SIDE is also better than SiNE, but no evidence of the benchmark between StEM and SIDE is traced. Consequently, to bridge this literature gap, 
this paper performs a detailed comparative analysis of these four signed network embedding methods, providing both a qualitative comparison based on these methods' theoretical characteristics as well as detailed quantitative analysis of their predictive performance for link sign prediction. To this end, three commonly used signed network datasets are used. As such, the main contributions of this paper are clear insights about the methods' performance both from predictive as well as run time perspective. Furthermore, it appears that StEM outperforms all other methods from both perspectives.

The remainder of this paper is organized as follows: Section II provides a brief overview of link sign prediction and representation learning; Section III introduces the state-ofthe-art signed network embedding methods and elaborates on the details of the each of the four benchmarked methods separately; Section IV provides a qualitative analysis while Section V starts with describing our experimental setup and datasets used and then presents extensive results including a hyperparameter sensitivity study. Finally, considerations for future work and a conclusion is given in VI.

\section{RELATED WORK}

Signed networks are getting more and more attention recently [9]-[12], [18]-[21] (for a more comprehensive overview of different tasks related to signed networks, see [20]). Among these, several studies address the problem of link sign prediction in particular [10], [18], [21]-[24]. A majority of studies on link sign prediction do not exploit representation learning but rather derive features from the network topology, or the interconnection between interaction semantics and the signs. For example, degree-based and triad-based features taking into account the sign of the link were used in [18]. In [10] the correlation between interactions and link signs is used together with a status and balance theory [15]. In [22], a latent factor model aimed at capturing the nature of interactions was proposed.

The network representation learning field expanded tremendously after the seminal work of [7]. However, most of the works, including [6], [7], are based on social theories (e.g. homophily and balance theory) or assumptions coming from social sciences [8], and are aimed for unsigned networks. As such, they are not suitable for signed link settings. Signed network embedding models tend to perform better than unsigned network embedding models as negative links provide added value over positive links in various analytical tasks, for instance, link prediction and node classification [9].

The focus of this work is on the studies of link sign prediction using representation learning, SNE [1], SiNE [2], SIDE [3] and StEM [4]. As with unsigned network representation learning, the methods used for learning representations can be categorized into matrix factorization, random walk and deep learning, as will be explained later in more details.

Besides negative links, also the link direction information in directed networks can give a significant performance boost in analytical tasks [31]. However, most methods only model the basic symmetric link structures (i.e. not directed).

\section{Signed Network Embedding Methods}

\section{A. Problem Formulation}

A signed network $G=(V, E)$ is an ordered pair of sets, where $V$ is the set of nodes and $E$ the set of edges. $E_{-}$denotes the set of negative edges and $E_{+}$the set of positive edges. That is $E_{-}=\left\{(u, v) \mid s_{u v}=-1\right\}$ and $E_{+}=\left\{(u, v) \mid s_{u v}=+1\right\}$. Each edge $e$ is represented as $(u, v)$ or $e_{u v}$, where $u$ is the source node and $v$ is the target node, and $s_{u v}$ denotes the sign of edge $(u, v)$ and it is set to 1 for positive and -1 for negative edges.

The goal of a node embedding is to learn a function $f: V \rightarrow R^{d}$ which maps each node $v \in V$ to a $d$-dimensional vector. The embedding function is parameterized by a $|V| \times d$ embedding matrix $W$ which consists of $d$-dimensional row vectors for each node $v$. In case of directed network, the node representation is defined as $\bar{W}_{v_{i}}=\left[W_{v_{i}}^{\text {in }}: W_{v_{i}}^{\text {out }}\right]$ where $W_{v_{i}}^{i n} \in R^{d}$ represents the source embedding of a node and $W_{v_{i}}^{\text {out }} \in R^{d}$ the target embedding of a node. In case of undirected network (or when the method does not incorporate link direction information), only a single node embedding is considered, denoted as $W_{v_{i}}$.

Signed link prediction aims to learn a function $g: E \rightarrow$ $\{+,-\}$ which assigns a label + or - to each edge $e \in E$. As such, it can be perceived as a binary classification problem on the edge level.

We will now briefly describe each of the four state-of-theart signed network embedding methods that are of our interest for benchmarking.

\section{B. SNE: Signed Network Embedding}

SNE [1] adopts the log-bilinear model [27], which is typically used in text settings to predict the next word given its predecessors. SNE can thus be considered as a maximum likelihood optimization problem, with the following objective function:

$$
J=\sum_{v \in V} \log p(v \mid h, q ; \theta)
$$

where $p(v \mid h, q ; \theta)$ is a conditional likelihood of target node $v$ given a path of nodes $h=\left[u_{1}, u_{2}, \ldots, u_{l}, v\right]$ and their edge types $q$, which are modeled with a softmax function:

$$
p(v \mid h, q ; \theta)=\frac{\exp (s(v, h))}{\sum_{v^{\prime} \in V} \exp \left(s\left(w^{\prime}, h\right)\right)}
$$

Here, $\theta=\left[w_{u_{i}}^{\text {in }}, w_{v}^{\text {out }}, c, b_{v}\right], w^{\prime}$ is the embedding of node $v^{\prime} \in V$ and $s(v, h)$ measures the similarity between predicted $\hat{w}_{h}$ and the actual representation $w_{v}^{\text {out }}$ of target node $v$ with $b_{v}$ being a bias term: $s(v, h)=\hat{w}_{h}^{T} w_{v}^{\text {out }}+b_{v}$.

Given a target node $v$ and a path $h=\left[u_{1}, u_{2}, \ldots, u_{l}, v\right]$, inferring a target node embedding $w_{v}^{\text {out }}$ is achieved by an element-wise multiplication (i.e. Hadamard product) between source embeddings $w_{u_{i}}^{i n}$ of all source nodes along the path $h$ and their position weight vector $c_{i}$ :

$$
\hat{w}_{h}=\sum_{i=1}^{l} c_{i} \otimes w_{u_{i}}^{i n}
$$




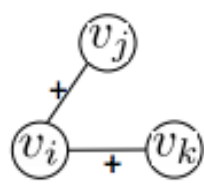

(a)

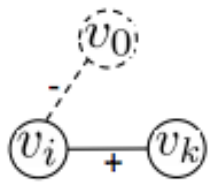

(b)

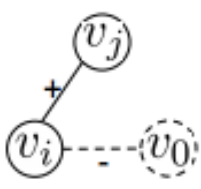

(c)
Fig. 1. An illustration of adding a virtual node. The original triplet (in a)) is divided into two new triplets as the virtual node $v_{0}$ will replace the nodes $v_{j}$ and $v_{k}$ in $\mathrm{b}$ ) and $\mathrm{c}$ ), respectively. Figure borrowed from [2].

The only adaptation from standard log-bilinear model is that $c_{i}$ here are signed-type vectors, representing the positive or negative edge, that is: $c_{i} \equiv c_{+} \in R^{d}$ if $s_{u_{i} u_{i+1}}=1$ and $c_{i} \equiv c_{-} \in R^{d}$ if $s_{u_{i} u_{i+1}}=-1$.

Additionally, SNE uses the sampled softmax approach from [29] instead of the regular softmax function in order to ease the computational difficulties.

\section{SiNE: Signed Network Embedding}

SiNE [2] is a deep learning based method which builds its objective function based on the extended structural balance theory, which assumes that the nodes in the network are more similar to other nodes with which it has positive links, than to those with whom it has negative links [13]. More formally, if we consider a triplet $\left(v_{i}, v_{j}, v_{k}\right)$ where $s_{i j}=$ $1, s_{i k}=-1, v_{i}, v_{j}, v_{k} \in V$, and if we denote by $f\left(w_{i}, w_{j}\right)$ a function which measures similarity between corresponding embeddings $w_{i}$ and $w_{j}$ of nodes $v_{i}$ and $v_{j}$, respectively, the extended structural balance theory assumes that $f\left(w_{i}, w_{j}\right) \geq$ $f\left(w_{i}, w_{k}\right)+\delta$, where $\delta$ is the similarity threshold (a large $\delta$ will force $v_{i}$ and $v_{j}$ closer and $v_{i}$ and $v_{k}$ further apart). Obviously, this theory has no effect in the cases of triplets with only positive or negative links. To accommodate to these cases, SiNE introduces virtual nodes with artificial links of different signs as illustrated in Figure 1. For the added virtual node $v_{0}$ and its embedding $w_{0}$, analogously as before, similarity can be modeled as: $f\left(w_{i}, w_{j}\right) \geq f\left(w_{i}, w_{0}\right)+\delta_{0}$, where $\delta_{0}$ is a threshold regulating the similarities.

Based on these, SiNE defines its objective function as:

$$
\begin{gathered}
\min _{W, w_{0}, \theta} \frac{1}{C}\left[\sum_{\left(w_{i}, w_{j}, w_{k}\right) \in P} \max \left(0, f\left(w_{i}, w_{k}\right)+\delta-f\left(w_{i}, w_{j}\right)\right)\right. \\
\left.+\sum_{\left(w_{i}, w_{j}, w_{0}\right) \in P_{0}} \max \left(0, f\left(w_{i}, w_{0}\right)+\delta_{0}-f\left(w_{i}, w_{j}\right)\right)\right] \\
+\lambda\left(\Re(\theta)+\|W\|_{F}^{2}+\left\|w_{0}\right\|_{2}^{2}\right)
\end{gathered}
$$

where $P$ and $P_{0}$ are two sets of triplets, $C=|P|+\left|P_{0}\right|$ is the size of the training data, $W=\left[w_{1}, w_{2}, \ldots, w_{n}\right]$ is the low-dimensional representation of all the nodes, $\theta$ is a set of parameters defining the similarity function $f$ and finally, $\mathfrak{R}(\theta)$ is a regularizer used to avoid overfitting and $\lambda$ is a parameter controlling the strength of that regularizer.

To optimize the given objective function, SiNE proposes considering $f$ as a non-linear function and using a deep learning framework which has as input the set of triplets. The optimization of the deep network is done by backpropagation, using a mini-batch stochastic gradient descent. A maximum of 300 training triplets per node is allowed as some nodes have a lot of connections and could, therefore, have a large number of triplets. The initialization of the deep network follows the approach in [30].

\section{SIDE: Signed Directed Network Embedding}

SIDE [3] is a method for learning node embeddings based on random walks and a neural language Skip-Gram model with negative sampling [5]. The objective function of SIDE is defined as:

$$
\begin{gathered}
J=\sum_{(u, v) \in D}\left[-\log P(u, v)+\sum_{j=1}^{n}-\log P\left(u, v_{j}^{\prime}\right)\right] \\
+\frac{\lambda}{2}\left(\left\|b^{\text {in, }+}\right\|^{2}+\left\|b^{\text {in,- }}\right\|^{2}+\left\|b^{\text {out },+}\right\|^{2}+\left\|b^{\text {out },-}\right\|^{2}\right)
\end{gathered}
$$

where $D \subseteq E$ is a set of edges evidenced in random walk corpus and likelihood $P(u, v)$ is defined as:

$$
P(u, v)=\left\{\begin{array}{l}
\sigma\left(W_{u}^{\text {out }} \cdot W_{v}^{\text {in }}+b_{u}^{\text {out },+}+b_{v}^{\text {in },+}\right), \text { if } s_{u v}>0 \\
\sigma\left(-W_{u}^{\text {out }} \cdot W_{v}^{\text {in }}+b_{u}^{\text {out },-}+b_{v}^{\text {in },-}\right), \text { if } s_{u v}<0 \\
\sigma\left(-W_{u}^{\text {out }} \cdot W_{v}^{\text {in }}\right), \text { if } v \text { is a noise }
\end{array}\right.
$$

while $(u, v)$ is a node pair in the random walk corpus; $v_{j}^{\prime} \in$ $N(u), N(u)$ represents the set of noise samples of node $u$; $b^{\text {in },+}, b^{\text {in,-}}, b^{\text {out },+}, b^{\text {out, },-}$ are the positive in-link, negative inlink, positive out-link and negative out-link bias, respectively; $W^{i n}$ and $W^{\text {out }}$ are in and out embedding matrices to be learnt; $s_{u v}$ is the aggregate sign of the path between nodes $u$ and $v$ (or simply the sign of the edge $(u, v)$ ) and finally, $\sigma$ is a sigmoid function. SIDE makes use of different social theories. First, it uses homophily [14] to extract co-occurence node pairs from the generated random walks. Additionally, based on the likelihood definition, embeddings will assign high similarity values for nodes $u, v$ with $s_{u v}=1$, and low similarity values otherwise. Second, it uses balance theory [15] to infer a sign of a link, assigning the sign to a multi-step connection to be negative if the number of negative edges along the path is odd, and positive otherwise. On the other hand, the direction is simply known by precedence in the path, as the random walk is bounded to follow the direction in the network. Third, bias terms $b^{\text {in/out },+/-}$ are used for modeling preferential attachment theory, as high bias terms lead to high likelihood of link formation and thus higher connectivity. Furthermore, given that bias terms are defined for each node and type of role in link formation, it allows for modeling personalized (i.e. per node) and asymmetric links.

Finally, gradient descent optimization is used to train the model with some additional optimization shortcuts to facilitate the learning process. 


\section{E. StEM: Signed neTwork Embedding Model}

StEM [4] is a deep learning method aiming at learning not only representations of nodes of different classes but also decision boundaries between opposing groups (e.g. friends and foes). Hence, on the contrary to the other methods, like SiNE, which is distance-based (thus, using only local information), StEM attempts to incorporate global information.

To this end, StEM starts from defining the task of learning the representation function as a maximum likelihood function problem:

$$
\min _{f, \theta} \sum_{(u, v) \in E}-\log P\left(y=\mathbb{I}_{\left\{s_{u v}=1\right\}} \mid u, v, \theta, f\right)
$$

where $s_{u v}$ is the sign of the edge between nodes $u$ and $v, \mathbb{I}$ is the indicator function, $f: V \rightarrow R^{d}$ is the representation function and $\theta$ is the set of parameters of the probability function. To ease the computation, $P$ is modeled as:

$$
P(x \mid u, v, \theta, f)= \begin{cases}\sigma(\mathcal{S}(u, v ; \theta, f)), & x=1 \\ 1-\sigma(\mathcal{S}(u, v ; \theta, f)), & x=0\end{cases}
$$

where $\sigma$ is the logit function, and the function $\mathcal{S}: V \times V \rightarrow R$ is defined as: $\mathcal{S}(u, v ; \theta, f)=\varphi\left(w_{u} ; \beta\right) M_{2} \varphi\left(w_{v} ; \beta\right)^{T}+b_{2}$ in order to play the role of node similarity function. Here, $w_{u}$ and $w_{v}$ are representations for $u$ and $v$, respectively; $M_{2} \in$ $R^{d \times d}$ is a learnable matrix of weights, $b_{2}$ is a learnable bias, $\varphi: R^{d} \rightarrow R^{d}$ is a parameterizable activation function, $\beta$ a learnable parameter of $\varphi$. In particular, the activation function $\varphi$ is a single-channeled parameterized leaky rectified linear unit as described in [32].

Again, a deep learning framework is proposed to learn the representation function while optimizing the objective function. More precisely, StEM designs a feed-forward neural network that takes two nodes, $u$ and $v$, as input and outputs the probability of a positive edge existing from $u$ and $v$. The representation for a node $u$ can be extracted from each forward pass through the network. Mini-batch back-propagation is used to train the network with undersampling during each epoch, thus ensuring that each batch has an equal number of positive and negative edges. Finally, the training algorithm applied is the AdaGrad method [28] and Xavier initialization [33] is used to set the parameters of the network prior to training.

\section{Qualitative Analysis}

The qualitative analysis presented in Table I, is based on the comparison of algorithmic characteristics of the four signed network embedding methods. In particular, it reports whether the method incorporates direction information, whether it uses random walks, what is the number of hyperparameters of the method and finally, what is its time complexity.

As mentioned, SiNE and StEM design their own deep learning framework and SNE and SIDE adapt the existing neural language models.

Noteworthy is the number of parameters that need to be set prior to learning the network embedding. More parameters could lead to more flexibility, but also a higher complexity and hence a more intensive fine-tuning process. More favorable would be a robust method with fewer parameters to tune.

Both SNE and SIDE have a rather high number of parameters to tune. Besides the two parameters, the embedding dimension size $d$ and a learning rate, which all methods have to set, SNE and SIDE have to tune additional random walk parameters: the number of walks per node $w$, the random walk length $l$ ) and a context size $L$, all used in their sampling approaches. Apart from these, SNE also requires the total number of samples to train, the number of classes to randomly sample per batch and the batch size, which is the number of training examples processed per step. SIDE's additional required parameters are the regularization parameter $\lambda$, the number of noise samples to form noise pairs and the batch size.

SiNE needs one parameter less than SNE and SIDE. The thresholds $\delta$ and $\delta_{0}$, the regularizer term $\lambda$, number of neural network layers $N$ and the number of epochs $t$ are the additional parameters SiNE has to set. StEM has only four parameters. Besides two common (the dimension size $d$ and learning rate), it only requires tuning of the number of epochs $t$ and a regularization term $\lambda$.

\section{Quantitative Analysis}

In this section, we first explain our experimental design and then we proceed with presenting results of link sign prediction and computational time as well as parameter sensitivity study.

\section{A. Experimental Design}

a) Data: The experiments are conducted on three signed networks. The first signed network, WikiEditor, is the only undirected network and was extracted from the UMD Wikipedia dataset [34]. The dataset is based on the co-edit relations, meaning that a positive edge is given between authors if the majority of their co-edits are from the same category and a negative edge when a majority of their coedits are of different categories. Slashdot is a technology news site where users are able to annotate other users as "friends" or "foes". The Slashdot dataset is a directed network based on whether a user annotates another user as a "friend" (i.e. positive edge) or as a "foe" (i.e. negative link). The last dataset, Epinions, is extracted from the Epinions website that is a product review site where users can form trust and distrust relationships with other users. Again, the dataset is built based on whether a user forms a trust relation (i.e. positive edge) or a distrust relation (i.e. negative edge) with another user. The statistics of the datasets are summarized in Table II.

b) Experimental Setup: The predictive performance of each method will be assessed by performing a link sign prediction task on every dataset. This means that we would like to predict the sign of several edges that are unobserved (i.e. test set) by the model trained on the other available edges (i.e. train set). For each signed network dataset its edges $E$ are partitioned into a training and test set. The training set was then used to learn representation using all four methods, SNE, SiNE, SIDE, and StEM. The learned source and target 
TABLE I

A GENERAL COMPARISON BETWEen SNE, SiNE, SIDE AND STEM. For TIME COMPLEXITY, $|V|$ DENOTES NUMBER OF NODES, $t$ NUMBER OF EPOCHS, $d$ NUMBER OF DIMENSIONS FOR THE EMBEDDING, $d_{n}, 1 \leq n \leq N$ IS NUMBER OF NODES IN THE $n^{\text {th }}$ LAYER OF NEURAL NETWORK, $S$ IS THE NUMBER OF TRAINING TRIPLETS, $\hat{e}=\min \left\{\left|E_{-}\right|,\left|E_{+}\right|\right\}$. SYMBOL “-” DENOTES THAT THE METHOD COMPLEXITY HAS NOT BEEN REPORTED IN THE ORIGINAL PAPER.

\begin{tabular}{|c||c|c|c|c|c|}
\hline Method & Considers sign & Considers direction & Random walk-based & \# of parameters & Time Complexity \\
\hline SNE & Yes & Yes & Yes & 8 & - \\
\hline SiNE & Yes & No & No & 7 & $\mathcal{O}\left(t S|V|\left(d d_{1}+\sum_{n=1}^{N} d_{i-1} d_{i}\right)\right)$ \\
\hline SIDE & Yes & Yes & Yes & 8 & $\mathcal{O}(|V|)$ \\
\hline StEM & Yes & No & No & 4 & $\mathcal{O}\left(t e \hat{e} d^{3}\right)$ \\
\hline
\end{tabular}

TABLE II

STATISTICS OF THE DATASETS

\begin{tabular}{|c|c|c|c|}
\hline & WikiEditor & Slashdot & Epinions \\
\hline \# of nodes & 21535 & 82140 & 131828 \\
\hline \# of edges & 348255 & 549202 & 841372 \\
\hline \# of + edges & $269251(77.3 \%)$ & $417943(76.1 \%)$ & $717690(85.3 \%)$ \\
\hline \# of - edges & $79004(22.7 \%)$ & $131259(23.9 \%)$ & $123682(14.7 \%)$ \\
\hline Type & Undirected & Directed & Directed \\
\hline
\end{tabular}

TABLE III

BINARY OPERATIONS FOR COMPOSING THE EDGE EMBEDDINGS FROM THE NODE EMBEDDINGS. HERE $w_{u}$ AND $w_{v}$ DENOTE EMBEDDINGS OF NODES $u$ AND $v$, RESPECTIVELY, WHILE $w_{u v}$ REPRESENTS EMBEDDING OF THE $\operatorname{EDGE}(u, v)$.

\begin{tabular}{|c|c|}
\hline Operation & Output \\
\hline Hadamard & $w_{u v}=w_{u} \times w_{v}$ \\
\hline Concatenation & $w_{u v}=w_{u} \otimes w_{v}$ \\
\hline L1-weight & $w_{u v}=\left|w_{u}-w_{v}\right|$ \\
\hline L2-weight & $w_{u v}=\left|w_{u}-w_{v}\right|^{2}$ \\
\hline Average & $w_{u v}=\frac{1}{2}\left(w_{u}+w_{v}\right)$ \\
\hline
\end{tabular}

node embedding are combined using binary operations in order to obtain edge features that are then used for training a simple logistic regression (LR) model, XGBoost (XGB) classifier and random forest (RF) classifier. Table III presents the different binary operations that were used for this purpose. The classifiers are then trained with these edge features in order to predict the edge signs from the edge representation of the test set. The random undersampling technique was used during the training of the classifiers due to the imbalance in $E$ of each signed network. Finally, the performance of the trained classifier is evaluated in terms of AUC ROC values using 5-fold cross-validation for each dataset.

All methods were trained with a learning rate set to 0.015 and a representation dimension $d$ of 64. For SNE and SIDE, the number of walks per node $w$ were set to 40 and the length of a random walk $l$ to 20 , and number of epochs $t$ is set to 100 for SiNE and StEM. Other parameters specific for each particular method were set as proposed in their original paper.

\section{B. Experimental Results on Link Sign Prediction}

As seen in Table IV, StEM outperforms the other methods on all datasets. SiNE underperforms on every dataset in comparison with the other methods, with the exception of Slashdot where SIDE has a slightly lower AUC value when using logistic regression. With respect to classifiers, except for WikiEditor, the best results are obtained using XGBoost.
For WikiEditor, Random Forests perform the best. Concatenation is almost always outperforming other binary operations, except for the following scenarios. First, for WikiEditor and logistic regression, L1-weight is a better choice for SiNE while Hadamard is better for the other methods. Second, SNE and StEM have better performance with the Hadamard operation for WikiEditor and XGBoost classifier.

It is worth nothing that these results could vary when using different parameters, such as length of walk $l$ and number of walks per node $w$ for SNE and SIDE. Currently, the choice of parameters has been made based on some basic fine-tuning and the values used in other studies. However, as StEM is parametrized only by few parameters, the results on StEM should be an overall good estimate of its performance.

\section{Parameter Sensitivity: Dimension Size}

We further evaluate how the dimension size of the node representation vectors affects the performance based on AUC. Each network embedding method is trained with dimensions $d$ set to $2,4,8,16,32,64,128,256$ and 512 and the performance of each embedding with each classifier are plotted in Figure 2. The AUC value for each method increases quickly with an increasing dimensions size, however each method seems to have a dimension threshold where the AUC value reaches its peak and decreases or evens out from thereon. This implies that significant increase in dimensions does not seem to pay off and provide a positive impact on the AUC score. It is worth nothing that having a higher dimension $d$ of node embeddings will come with a larger amount of required storage capacity as well.

However, keeping the embedding dimension $d$ rather small $(<8)$ apparently does not tend to be beneficial either, as the learned network embeddings end up not being able to include all relevant information on the topology and link structure of the network. As the embedding dimension $d$ is getting larger, most of the relevant information is captured.

\section{Computation time}

The computation time of each signed network embedding method is also of importance when considering to learn a representation of a network. A comparison is made between the run times of the four methods for learning a network embedding of each dataset. Following parameters were used: both a dimension size $d$ of 8 and 64, number of epochs $t$ set to 100 for StEM and SiNE, and number of walks per node $w$ set to 40 , length of random walk $l$ set to 20 for SNE and 
TABLE IV

PERFORMANCE BASED ON MEAN AUC FROM 5-FOLD CROSS-VALIDATION ON LINK SIGN PREDICTION FOR EACH METHOD AND EACH BINARY OPERATOR WITH DIMENSIONS $d=64$. BEST RESULTS PER CLASSIFIER, DATASET AND METHOD ARE DISPLAYED IN BOLDFACE. BEST RESULTS PER DATASET ARE IN BOLDFACE AND UNDERLINED.

\begin{tabular}{|c|c|c|c|c|c|c|c|}
\hline \multirow{2}{*}{ Classifier } & \multirow{2}{*}{ Dataset } & \multirow{2}{*}{ Method } & \multicolumn{5}{|c|}{ Operator } \\
\hline & & & Concatenation & Hadamard & L1-weight & L2-weight & Average \\
\hline \multirow{12}{*}{ LR } & \multirow{4}{*}{ WikiEditor } & SNE & 0.855 & 0.976 & 0.935 & 0.945 & 0.788 \\
\hline & & SiNE & 0.743 & 0.648 & 0.755 & 0.722 & 0.727 \\
\hline & & SIDE & 0.865 & 0.907 & 0.765 & 0.750 & 0.850 \\
\hline & & StEM & 0.908 & 0.937 & 0.919 & 0.920 & 0.907 \\
\hline & \multirow{4}{*}{ Slashdot } & SNE & 0.800 & 0.740 & 0.694 & 0.698 & 0.751 \\
\hline & & SiNE & 0.722 & 0.593 & 0.673 & 0.646 & 0.629 \\
\hline & & SIDE & 0.706 & 0.657 & 0.645 & 0.639 & 0.657 \\
\hline & & StEM & 0.893 & 0.877 & 0.729 & 0.756 & 0.879 \\
\hline & \multirow{4}{*}{ Epinions } & SNE & 0.901 & 0.857 & 0.816 & 0.816 & 0.851 \\
\hline & & SiNE & 0.854 & 0.720 & 0.784 & 0.762 & 0.633 \\
\hline & & SIDE & 0.865 & 0.852 & 0.804 & 0.806 & 0.858 \\
\hline & & StEM & 0.936 & 0.904 & 0.806 & 0.842 & 0.886 \\
\hline \multirow{12}{*}{ XGB } & \multirow{4}{*}{ WikiEditor } & SNE & 0.972 & 0.977 & 0.955 & 0.955 & 0.968 \\
\hline & & SiNE & 0.837 & 0.674 & 0.693 & 0.692 & 0.731 \\
\hline & & SIDE & 0.963 & 0.958 & 0.912 & 0.912 & 0.936 \\
\hline & & StEM & 0.976 & 0.980 & 0.948 & 0.948 & 0.959 \\
\hline & \multirow{4}{*}{ Slashdot } & SNE & 0.841 & 0.769 & 0.705 & 0.705 & 0.773 \\
\hline & & SiNE & 0.753 & 0.682 & 0.701 & 0.701 & 0.702 \\
\hline & & SIDE & 0.791 & 0.704 & 0.699 & 0.699 & 0.729 \\
\hline & & StEM & $\underline{0.949}$ & 0.933 & 0.747 & 0.747 & 0.915 \\
\hline & \multirow{4}{*}{ Epinions } & SNE & $\overline{\mathbf{0 . 9 3 1}}$ & 0.877 & 0.832 & 0.832 & 0.882 \\
\hline & & SiNE & 0.903 & 0.768 & 0.813 & 0.813 & 0.807 \\
\hline & & SIDE & 0.932 & 0.890 & 0.865 & 0.865 & 0.902 \\
\hline & & StEM & $\underline{0.988}$ & 0.963 & 0.851 & 0.851 & 0.925 \\
\hline \multirow{12}{*}{$\mathbf{R F}$} & \multirow{4}{*}{ WikiEditor } & SNE & $\overline{\mathbf{0 . 9 7 8}}$ & 0.974 & 0.956 & 0.960 & 0.971 \\
\hline & & SiNE & 0.891 & 0.798 & 0.744 & 0.745 & 0.768 \\
\hline & & SIDE & 0.977 & 0.969 & 0.956 & 0.956 & 0.963 \\
\hline & & StEM & $\underline{0.981}$ & 0.979 & 0.939 & 0.939 & 0.953 \\
\hline & \multirow{4}{*}{ Slashdot } & SNE & $\overline{0.854}$ & 0.779 & 0.689 & 0.687 & 0.723 \\
\hline & & SiNE & 0.757 & 0.701 & 0.716 & 0.715 & 0.723 \\
\hline & & SIDE & 0.838 & 0.726 & 0.726 & 0.724 & 0.750 \\
\hline & & StEM & 0.939 & 0.924 & 0.776 & 0.776 & 0.903 \\
\hline & \multirow{4}{*}{ Epinions } & SNE & 0.942 & 0.890 & 0.820 & 0.821 & 0.877 \\
\hline & & SiNE & 0.877 & 0.820 & 0.812 & 0.813 & 0.820 \\
\hline & & SIDE & 0.946 & 0.911 & 0.890 & 0.890 & 0.917 \\
\hline & & StEM & 0.983 & 0.959 & 0.860 & 0.861 & 0.918 \\
\hline
\end{tabular}

SIDE. Other parameters remain the same as in the previous experiments (i.e. link sign prediction). Table $\mathrm{V}$ shows the times recorded for every method on each dataset for a dimension size $d$ of 8 and 64. The device used for training has the following specifications: Intel Core i9 $2.9 \mathrm{GHz}$ CPU with $32 \mathrm{~GB}$ RAM and a GeForce GTX1050 Ti graphics card as GPU.

Firstly, creating the random walks takes time and can increase based on the size of the dataset, number of walks per node $w$ and length of random walk $l$. The time it takes to create the random walks is therefore included in the training runtime of SNE and SIDE. Secondly, StEM outperforms every method based on runtime, and this with a noteworthy difference. Whereas SIDE performs decent on the smaller datasets (i.e. WikiEditor and Slashdot), but performs poorly on the largest dataset (i.e. Epinions). Finally, both SNE and SiNE have tolerable run times for each dataset, however SiNE has much better results for both WikiEditor and Slashdot.

\section{CONCLUSION}

Signed networks, that is, networks which incorporate sign information of edges, are useful for many different applications. Therefore, featurizing these kind of networks with
TABLE V

RUN TIME FOR EACH METHOD TRAINED ON THE DIFFERENT DATASETS WITH A DIMENSION $d$ OF 8 AND 64. BEST RESULTS PER DIMENSION AND DATASET ARE DENOTED IN BOLDFACE.

\begin{tabular}{|c|c|c|c|c|c|}
\hline \multirow{2}{*}{ Dimension } & \multirow{2}{*}{ Dataset } & \multicolumn{4}{|c|}{ Method } \\
\cline { 3 - 6 } & & SNE & SiNE & SIDE & StEM \\
\hline \multirow{3}{*}{8} & WikiEditor & $11 \mathrm{~m} 41 \mathrm{~s}$ & $3 \mathrm{~m} 16 \mathrm{~s}$ & $10 \mathrm{~m} 32 \mathrm{~s}$ & $\mathbf{4 8 s}$ \\
\cline { 2 - 6 } & Slashdot & $19 \mathrm{~m} 54 \mathrm{~s}$ & $2 \mathrm{~m} 40 \mathrm{~s}$ & $25 \mathrm{~m} 5 \mathrm{~s}$ & $\mathbf{1 m 1 1}$ \\
\cline { 2 - 6 } & Epinions & $24 \mathrm{~m} 34 \mathrm{~s}$ & $20 \mathrm{~m} 3 \mathrm{~s}$ & $58 \mathrm{~m} 57 \mathrm{~s}$ & $\mathbf{1 m 2 9}$ \\
\hline \multirow{3}{*}{64} & WikiEditor & $13 \mathrm{~m} 14 \mathrm{~s}$ & $3 \mathrm{~m} 18 \mathrm{~s}$ & $10 \mathrm{~m} 48 \mathrm{~s}$ & $\mathbf{1 m 2 1}$ \\
\cline { 2 - 6 } & Slashdot & $20 \mathrm{~m} 29 \mathrm{~s}$ & $2 \mathrm{~m} 46 \mathrm{~s}$ & $26 \mathrm{~m} 56 \mathrm{~s}$ & $\mathbf{1 m 5 6 s}$ \\
\cline { 2 - 6 } & Epinions & $25 \mathrm{~m} 6 \mathrm{~s}$ & $20 \mathrm{~m} 46 \mathrm{~s}$ & $1 \mathrm{~h} 0 \mathrm{~m} 51 \mathrm{~s}$ & $\mathbf{2 m 1 3 s}$ \\
\hline
\end{tabular}

representation learning is at least as relevant as in the case of unsigned networks. While several signed network representation learning techniques have already been proposed, current literature lacks the comparison of these methods and clear insights on their performance. Consequently, this paper performs a benchmarking of four state-of-the-art signed network embedding methods, known as SNE, SiNE, SIDE and StEM. First, these methods are qualitatively compared based on their theoretical foundations. Next, an extensive quantitative 

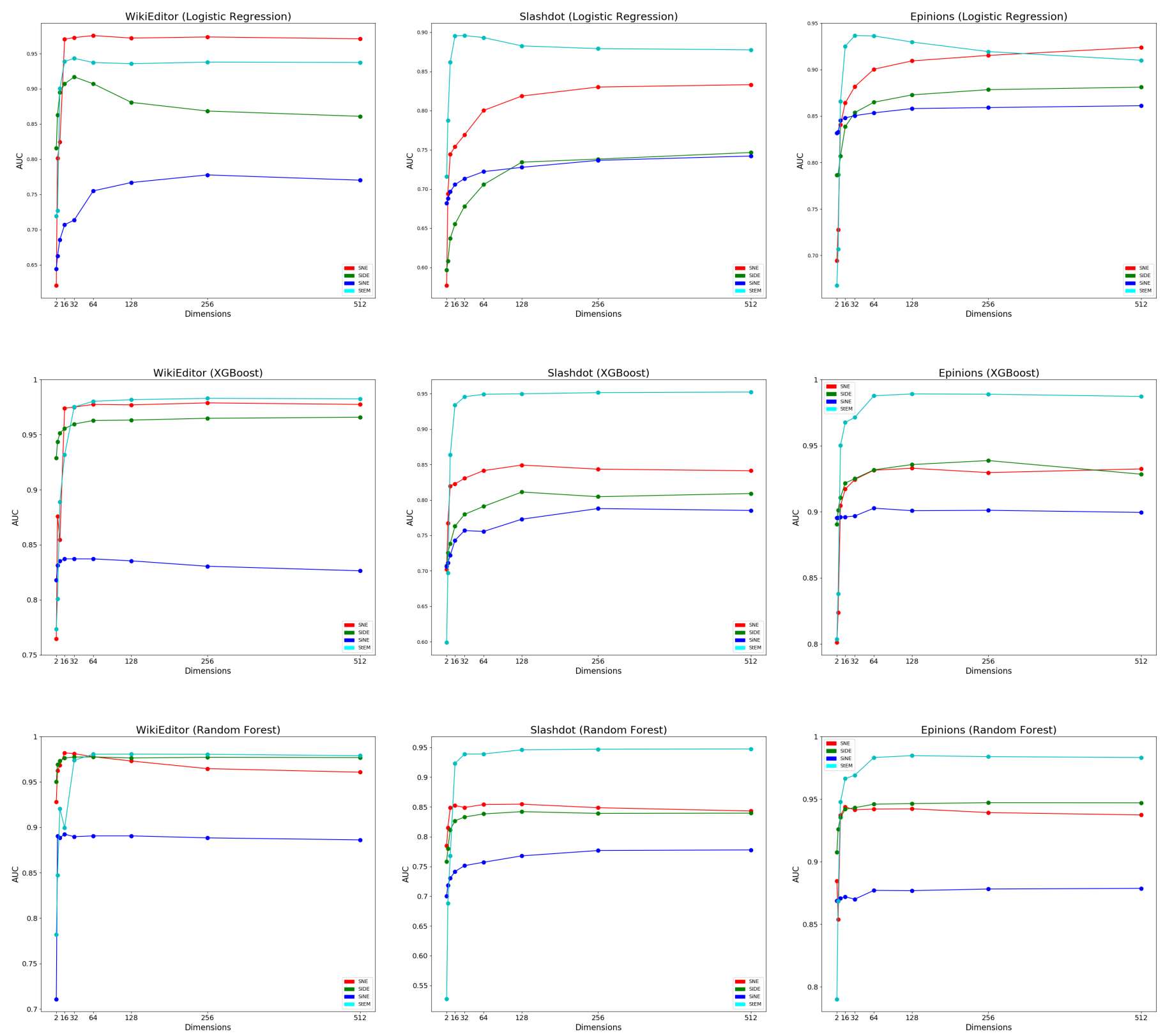

Fig. 2. Performance based on mean AUC from 5-fold cross-validation on link sign prediction with dimension $d$ of 2, 4, 8, 16, 32, 64, 128,256 and 512.

analysis is performed for the link sign prediction problem on several signed networks. Results of the conducted experiments have shown that most of these open-source methods are able to learn useful representations which perform well on the particular problem of link sign prediction. More specifically, the experiments showcased that the StEM method outperforms the other methods and could, therefore, be an excellent choice when considering further extensions of signed network embedding methods.

This being said, we are aware of several current limitations and, consequently, possibilities for future work.

First, a more extensive parameter tuning could lead to a better performance for each method. Second, a more general benchmark could be obtained by testing the performance of each of the considered methods on several other machine learning tasks e.g., node classification, link prediction or data visualization. Third, the benchmark could be extended or used for comparison with several other signed network embedding methods. Other promising methods are, for instance, SGCN [34] and DNE-SBP [35]. Finally, studying the sensitivity of other parameters (and not only the embedding dimension size d) could also offer important insights. 


\section{REFERENCES}

[1] Yuan, S., Wu, X., \& Xiang, Y. (2017, May). SNE: signed network embedding. In Pacific-Asia conference on knowledge discovery and data mining (pp. 183-195). Springer, Cham.

[2] Wang, S., Tang, J., Aggarwal, C., Chang, Y., \& Liu, H. (2017, June) Signed network embedding in social media. In Proceedings of the 2017 SIAM international conference on data mining (pp. 327-335). Society for Industrial and Applied Mathematics.

[3] Kim, J., Park, H., Lee, J. E., \& Kang, U. (2018, April). Side: Representation learning in signed directed networks. In Proceedings of the 2018 World Wide Web Conference on World Wide Web (pp. 509-518). International World Wide Web Conferences Steering Committee.

[4] Rahaman, I., \& Hosein, P. 2018. A method for learning representation of signed networks.

[5] Mikolov, T., Chen, K., Corrado, G., \& Dean, J. (2013). Efficient estimation of word representations in vector space. arXiv preprint arXiv:1301.3781.

[6] Grover, A., \& Leskovec, J. (2016, August). node2vec: Scalable feature learning for networks. In Proceedings of the 22nd ACM SIGKDD international conference on Knowledge discovery and data mining (pp. 855-864). ACM.

[7] Perozzi, B., Al-Rfou, R., \& Skiena, S. (2014, August). Deepwalk: Online learning of social representations. In Proceedings of the 20th ACM SIGKDD international conference on Knowledge discovery and data mining (pp. 701-710). ACM.

[8] Tang, J., Chang, Y., \& Liu, H. (2014). Mining social media with social theories: a survey. ACM Sigkdd Explorations Newsletter, 15(2), 20-29.

[9] Kunegis, J., Preusse, J., \& Schwagereit, F. (2013, May). What is the added value of negative links in online social networks?. In Proceedings of the 22nd international conference on World Wide Web (pp. 727-736). ACM.

[10] Tang, J., Chang, S., Aggarwal, C., \& Liu, H. (2015, February). Negative link prediction in social media. In Proceedings of the eighth ACM international conference on web search and data mining (pp. 87-96). ACM.

[11] Leskovec, J., Huttenlocher, D., \& Kleinberg, J. (2010, April). Signed networks in social media. In Proceedings of the SIGCHI conference on human factors in computing systems (pp. 1361-1370). ACM.

[12] Kunegis, J., Lommatzsch, A., \& Bauckhage, C. (2009, April). The slashdot zoo: mining a social network with negative edges. In Proceedings of the 18th international conference on World wide web (pp. 741-750). ACM.

[13] Cygan, M., Pilipczuk, M., Pilipczuk, M., \& Wojtaszczyk, J. O. (2012, August). Sitting closer to friends than enemies, revisited. In International Symposium on Mathematical Foundations of Computer Science (pp. 296307). Springer, Berlin, Heidelberg.

[14] McPherson, M., Smith-Lovin, L., \& Cook, J. M. (2001). Birds of a feather: Homophily in social networks. Annual review of sociology, 27(1), 415-444.

[15] Cartwright, D., \& Harary, F. (1956). Structural balance: a generalization of Heider's theory. Psychological review, 63(5), 277.

[16] Hamilton, W. L., Ying, R., \& Leskovec, J. (2017). Representation learning on graphs: Methods and applications. arXiv preprint arXiv:1709.05584.

[17] Bengio, Y., Courville, A., \& Vincent, P. (2013). Representation learning: A review and new perspectives. IEEE transactions on pattern analysis and machine intelligence, 35(8), 1798-1828.

[18] Leskovec, J., Huttenlocher, D., \& Kleinberg, J. (2010, April). Predicting positive and negative links in online social networks. In Proceedings of the 19th international conference on World wide web (pp. 641-650). ACM.

[19] Brzozowski, M. J., Hogg, T., \& Szabo, G. (2008, April). Friends and foes: ideological social networking. In Proceedings of the SIGCHI conference on human factors in computing systems (pp. 817-820). ACM.

[20] Tang, J., Chang, Y., Aggarwal, C., \& Liu, H. (2016). A survey of signed network mining in social media. ACM Computing Surveys (CSUR), 49(3), 42 .

[21] Guha, R., Kumar, R., Raghavan, P., \& Tomkins, A. (2004, May). Propagation of trust and distrust. In Proceedings of the 13th international conference on World Wide Web (pp. 403-412). ACM.

[22] Yang, S. H., Smola, A. J., Long, B., Zha, H., \& Chang, Y. (2012, August). Friend or frenemy?: predicting signed ties in social networks. In Proceedings of the 35th international ACM SIGIR conference on Research and development in information retrieval (pp. 555-564). ACM.
[23] Yu, X., \& Xie, J. (2014). Modeling mutual influence between social actions and social ties. In Proceedings of COLING 2014, the 25th International Conference on Computational Linguistics: Technical Papers (pp. 848-859).

[24] Ye, J., Cheng, H., Zhu, Z., \& Chen, M. (2013, May). Predicting positive and negative links in signed social networks by transfer learning. In Proceedings of the 22nd international conference on World Wide Web (pp. 1477-1488). ACM.

[25] Newman, M. E. (2005). A measure of betweenness centrality based on random walks. Social networks, 27(1), 39-54.

[26] Lovsz, L. (1993). Random walks on graphs: A survey. Combinatorics, Paul erdos is eighty, 2(1), 1-46.

[27] Mnih, A., \& Hinton, G. E. (2009). A scalable hierarchical distributed language model. In Advances in neural information processing systems (pp. 1081-1088).

[28] J. Duchi, E. Hazan, Y. Singer. Duchi, J., Hazan, E., \& Singer, Y. (2011). Adaptive subgradient methods for online learning and stochastic optimization. Journal of Machine Learning Research, 12(Jul), 2121-2159.

[29] Cygan, M., Pilipczuk, M., Pilipczuk, M., \& Wojtaszczyk, J. O. (2012, August). Sitting closer to friends than enemies, revisited. In International Symposium on Mathematical Foundations of Computer Science (pp. 296307). Springer, Berlin, Heidelberg.

[30] Lian, W., Henao, R., Rao, V., Lucas, J., \& Carin, L. (2015, June). A multitask point process predictive model. In International Conference on Machine Learning (pp. 2030-2038).

[31] Golder, S. A., \& Yardi, S. (2010, August). Structural predictors of tie formation in twitter: Transitivity and mutuality. In 2010 IEEE Second International Conference on Social Computing (pp. 88-95). IEEE.

[32] He, K., Zhang, X., Ren, S., \& Sun, J. (2015). Delving deep into rectifiers: Surpassing human-level performance on imagenet classification. In Proceedings of the IEEE international conference on computer vision (pp. 1026-1034).

[33] Kumar, S., Spezzano, F., \& Subrahmanian, V. S. (2015, August). Vews: A wikipedia vandal early warning system. In Proceedings of the 21th ACM SIGKDD international conference on knowledge discovery and data mining (pp. 607-616). ACM.

[34] Derr, T., Ma, Y., \& Tang, J. (2018, November). Signed graph convolutional networks. In 2018 IEEE International Conference on Data Mining (ICDM) (pp. 929-934). IEEE.

[35] Shen, X., \& Chung, F. L. (2018). Deep network embedding for graph representation learning in signed networks. IEEE transactions on cybernetics.

[36] Heider, F. (1946). Attitudes and cognitive organization. The Journal of psychology, 21(1), 107-112. 